\title{
Gradient Elasticity Formulations for Micro/Nanoshells
}

\author{
Bohua Sun ${ }^{1}$ and E. C. Aifantis ${ }^{2,3,4}$ \\ ${ }^{1}$ Centre for Mechanics and Technology, Faculty of Engineering, Cape Peninsula University of Technology, \\ Cape Town, South Africa \\ ${ }^{2}$ Lab of Mechanics and Materials, Polytechnic School, Aristotle University, 54124 Thessaloniki, Greece \\ ${ }^{3}$ Michigan Technological University, Houghton, MI 49931, USA \\ ${ }^{4}$ King Abdulaziz University, Jeddah 21589, Saudi Arabia \\ Correspondence should be addressed to Bohua Sun; sunb@cput.ac.za
}

Received 4 October 2013; Accepted 23 January 2014; Published 20 February 2014

Academic Editor: Razali Ismail

Copyright (C) 2014 B. Sun and E. C. Aifantis. This is an open access article distributed under the Creative Commons Attribution License, which permits unrestricted use, distribution, and reproduction in any medium, provided the original work is properly cited.

The focus of this paper is on illustrating how to extend the second author's gradient theory of elasticity to shells. Three formulations are presented based on the implicit gradient elasticity constitutive relation $\left(1-l_{d}^{2} \nabla^{2}\right) \sigma_{i j}=C_{i j k l}\left(1-l_{s}^{2} \nabla^{2}\right) \varepsilon_{k l}$ and its two approximations $\left(1+l_{s}^{2} \nabla^{2}-l_{d}^{2} \nabla^{2}\right) \sigma_{i j}=C_{i j k l} \varepsilon_{k l}$ and $\sigma_{i j}=C_{i j k l}\left(1+l_{d}^{2} \nabla^{2}-l_{s}^{2} \nabla^{2}\right) \varepsilon_{k l}$.

\section{Introduction}

Many unexpected applications were found due to superior thermochemomechanical and optoelectromagnetic material properties noted at the nanoscale. Nanoscale structures, such as nanobeams, nanoplates, and nanoshells, were used in many MEMS and NEMS applications. Therefore, understanding the static and dynamic behaviour of them is important for reliable design of micro- and nanodevices.

Experimental studies are generally difficult at the nanoscale due to resolution limitation of available nanoprobes. Molecular dynamics (MD) experiments were therefore normally employed to understand nanoscale behavior. Unfortunately, MD studies are limited to small number of atoms and short time intervals. Continuum models were then proposed as an alternative solution method.

For mechanical behaviour modelling of nanostructures, the classical continuum mechanics models are not adequate because these models only contain bulk material properties and cannot capture inhomogeneously evolving microstructures and related size effects. To simulate nanostructures, a number of continuum theories have been used to predict the influence of nanoscale effects, such as couple stress and
Cosserat theories, nonlocal elasticity, and gradient elasticity. The gradient theory is an extension of classical theory to include additional higher-order spatial derivatives of strain and/or stress, as well as (internal) acceleration. It has been shown to be a powerful alternative tool for dealing with nanostructures without resorting to expensive MD computations.

The use of gradient elasticity to simulate the mechanical behaviour of materials and structures is not a novel ideain fact, it has been advocated more than a century and a half ago; however, the scope has varied widely over the years. Pioneer work was done by Cauchy in 1850s [1, 2]. Cauchy suggested the use of higher-order spatial derivatives in the continuum equations describing elastic properties in order to approximate the behaviour of discrete lattice models with more accuracy, whereby the size of the elementary volume appeared as an additional constitutive parameter. As a followup of Cauchy's initial work on the subject, one may consider Cosserat's theory developed half a century later (early 1900s) [3]. Mindlin's [4, 5] strain gradient theory of elasticity involving five extra phenomenological constants should be mentioned, as reference to it is being made by researchers today. In modern times, Eringen [6-9] derived 
a simple stress-gradient elasticity model from his earlier integral nonlocal theory, but interest in this work has remained largely dormant till the late 1990s. In early 1990s, Aifantis [10] proposed a robust strain gradient theory of elasticity, which has led to notably straightforward finite element implementations. This has been generalized in Aifantis $[11,12]$ to include both strain and stress gradients, thus incorporating the effects of both Eringen's and Aifantis' ingredients of gradient theories. For details, the reader should consult the articles listed in the first six references provided in the bibliography, as well as in Eringen's book [9] also listed there. In the last reference, an initial effort by the first author to apply gradient theory to nanoshells and nanoplates is outlined.

The key issue of formulations of gradient shell theory is how to fit the new gradient constitution equation into the general framework of shell theory. In this short paper, we will restrict ourselves only on that issue.

\section{Linear Shell Theories Formulation [13]}

The most common shell theories are those based on linear elasticity concepts. Linear shell theories predict adequately stresses and deformations for shells exhibiting small elastic deformations; that is, deformations for which it is assumed that the equilibrium equation conditions for deformed shell surfaces are the same as if they were not deformed, and Hooke's law applies. For the purpose of analysis, a shell may be considered as a three-dimensional body, and the methods of the theory of linear elasticity may then be applied. However, a calculation based on these methods will generally be very difficult and complicated. In the theory of shells, an alternative simplified method is therefore employed. According to this method and adapting some hypotheses, the $3 \mathrm{D}$ problem of shell equilibrium and straining may be reduced to the analysis of its middle surface only; that is, the given shell, as discussed earlier as a thin plate, may be regarded as some 2D body. In the development of thin shell theories, simplification is accomplished by reducing the shell problems to the study of deformations of the middle surface.

Shell theories of varying degrees of accuracy were derived, depending on the degree to which the elasticity equations were simplified. The approximations necessary for the development of an adequate theory of shells have been the subject of considerable discussions among investigators in the field. We present below a brief outline of elastic shell theories in an historical context.

Love [14] was the first investigator to present a successful approximation shell theory based on classical linear elasticity. To simplify the strain-displacement relationships and, consequently, the constitutive relations, Love applied, to the shell theory, the Kirchhoff hypotheses developed originally for the plate bending theory, together with the small deflection and thinness of the shell assumptions. This set of assumptions is commonly called the Kirchhoff-Love assumptions. The Love theory of thin elastic shells is also referred to as the firstorder approximation shell theory. In spite of its popularity and common character, Love's theory was not free from some deficiencies, including its inconsistent treatment of small terms, where some were retained and others were rejected, although they were of the same order. This meant that, for certain shells, Love's differential operator matrix on the displacements, in the equations of equilibrium, became unsymmetric. Obviously, this violated Betti's theorem of reciprocity. Love's theory [14] also contained some other inconsistencies. The need for a mathematically rigorous two-dimensional set of the shell equations employing the Kirchhoff-Love assumptions led to different versions of the first order approximation theories.

The middle surface of the shell is determined by the parametric representation $\mathbf{r}=\mathbf{r}\left(\theta^{1}, \theta^{2}\right)$. The covariant base vectors of the middle surface are defined by $\mathbf{a}_{\alpha}=\mathbf{r}_{, \alpha}$, the comma notation being used to denotes partial derivative with respect to the parameters. The metric tensors of a surface are defined by $a_{\alpha \beta}=\mathbf{a}_{\alpha} \cdot \mathbf{a}_{\beta}=a_{\beta \alpha}, a^{\alpha \lambda} a_{\lambda \beta}=\delta_{\beta}^{\alpha}$, where $a_{\alpha \beta}$ and $a^{\alpha \beta}$ are covariant and contravariant symmetric tensors of order two, respectively. The contravariant base vectors of a surface are given by $\mathbf{a}^{\alpha}=a^{\alpha \beta} \mathbf{a}_{\beta}$ and the first fundamental forms of the metric tensors are given by $\mathbf{a}^{\alpha} \cdot \mathbf{a}^{\beta}=a^{\alpha \beta}, \mathbf{a}^{\alpha} \cdot \mathbf{a}_{\beta}=\delta_{\beta}^{\alpha}$. Defining $\mathbf{a}_{3}=(1 / \sqrt{a}) \mathbf{a}_{1} \times \mathbf{a}_{2}$, we have the relations $\mathbf{a}_{3} \cdot \mathbf{a}_{\alpha}=0, \mathbf{a}_{3} \cdot \mathbf{a}^{\alpha}=0$. The permutation tensors $e_{\alpha \beta}$ and $e^{\alpha \beta}$ are defined by $e_{\alpha \beta}: e_{12}=$ $-e_{21}=\sqrt{a}, e_{\alpha \beta}=-e_{\beta \alpha}, e^{\alpha \beta}=-e^{\beta \alpha}, e_{11}=e_{22}=0$ and $e^{\alpha \beta}$ : $e^{12}=-e^{21}=1 / \sqrt{a}, e^{11}=e^{22}=0$. The following expressions are then obtained for the vector products of the base vectors: $\mathbf{a}_{\alpha} \times \mathbf{a}_{\beta}=e_{\alpha \beta} \mathbf{a}_{3}, \mathbf{a}^{\alpha} \times \mathbf{a}^{\beta}=e^{\alpha \beta} \mathbf{a}_{3}, \mathbf{a}_{3} \times \mathbf{a}_{\alpha}=e_{\alpha \beta} \mathbf{a}^{\beta}, \mathbf{a}_{3} \times \mathbf{a}^{\alpha}=$ $e^{\alpha \beta} \mathbf{a}_{\beta}$. The coefficients of the second fundamental form are $b_{\alpha \beta}=b_{\beta \alpha}=\mathbf{a}_{\alpha, \beta} \cdot \mathbf{a}_{3}=-\mathbf{a}_{3, \alpha} \cdot \mathbf{a}_{\beta}$. Then the derivatives of the base vectors are determined by $\mathbf{a}_{\alpha, \beta}=\mathbf{a}_{\beta, \alpha}=\left\{\begin{array}{c}\lambda \\ \alpha \beta\end{array}\right\} \mathbf{a}_{\lambda}+b_{\alpha \beta} \mathbf{a}_{3}$, $\mathbf{a}_{3, \alpha}=-b_{\alpha}^{\beta} \mathbf{a}_{\beta}, \mathbf{a}_{, \beta}^{\alpha}=\mathbf{a}_{\beta, \alpha}=-\left\{\lambda_{\lambda}^{\alpha}\right\} \mathbf{a}^{\lambda}+b_{\beta}^{\alpha} \mathbf{a}_{3}$. The Christoffel symbols of the second kind for the surface are defined by the familiar relationships $\left\{\begin{array}{c}\lambda \\ \alpha \beta\end{array}\right\}=\mathbf{a}_{\alpha, \beta} \cdot \mathbf{a}^{\lambda}=-\mathbf{a}_{, \beta}^{\lambda} \cdot \mathbf{a}_{\alpha}=-\mathbf{a}_{, \alpha}^{\lambda} \cdot \mathbf{a}_{\beta}$, which can be expressed in the following manner in terms of the metric tensor $\left\{\begin{array}{c}\lambda \\ \alpha \beta\end{array}\right\}=a^{\gamma \lambda}(1 / 2)\left(a_{\beta \lambda, \alpha}+a_{\lambda \alpha, \beta}-a_{\alpha \beta, \lambda}\right)$.

The displacement vector $\mathbf{v}\left(\theta^{1}, \theta^{2}\right)$ is resolved in the directions of the base vectors $\mathbf{a}^{\alpha}$ and the normal vector $\mathbf{a}_{3}$, so that $\mathbf{v}=v_{\beta} \mathbf{a}^{\beta}+v_{3} \mathbf{a}_{3}$. The strain measures of the middle surface of the shell is defined by $\varepsilon_{\alpha \beta}=(1 / 2)\left(a_{\alpha \beta}^{*}-a_{\alpha \beta}\right)_{\text {lin }}=(1 / 2) \delta a_{\alpha \beta}$. As the difference $a_{\alpha \beta}^{*}-a_{\alpha \beta}$ between the metric tensors in the deformed state and in the reference state is again a tensor, we obtain $\varepsilon_{\alpha \beta}=(1 / 2)\left(\mathbf{a}_{\alpha} \cdot \mathbf{v}_{, \beta}+\mathbf{a}_{\beta} \cdot \mathbf{v}_{, \alpha}\right)$, which expresses the strain tensor in terms of the displacement components. The derivative of the displacement vector is written as $\mathbf{v}_{, \alpha}=$ $\varepsilon_{\alpha \beta} \mathbf{a}^{\beta}+\boldsymbol{\omega} \times \mathbf{a}_{\alpha}$, where $\boldsymbol{\omega}=\omega_{\alpha} \widehat{\mathbf{a}}^{\alpha}+\omega_{3} \mathbf{a}_{3}, \omega_{\alpha}=-\left(v_{3, \alpha}+b_{\alpha}^{\beta} v_{\beta}\right)$, $\omega_{3}=(1 / 2) e^{\alpha \beta} v_{\beta \mid \alpha}$, giving the components of the rotation vector in terms of the displacements. The bending tensor $\kappa_{\alpha \beta}$ can be expressed in terms of the displacement components through the following expression:

$$
\begin{gathered}
\kappa_{\alpha \beta}=-\left[v_{3 \mid \alpha \beta}+b_{\alpha \mid \beta}^{\lambda} v_{\lambda}+\frac{3}{4}\left(b_{\alpha}^{\lambda} v_{\lambda \mid \beta}+b_{\beta}^{\lambda} v_{\lambda \mid \alpha}\right)\right. \\
\left.-\frac{1}{4}\left(b_{\alpha}^{\lambda} v_{\beta \mid \lambda}+b_{\beta}^{\lambda} v_{\alpha \mid \lambda}\right)\right]
\end{gathered}
$$


which along with

$$
\begin{gathered}
e^{\alpha \beta}\left[\kappa_{\alpha \lambda \mid \beta}+\frac{1}{2}\left(b_{\alpha}^{\lambda} \varepsilon_{\lambda \gamma}-b_{\gamma}^{\lambda} \varepsilon_{\lambda \alpha}\right)_{\mid \beta}+b_{\alpha}^{\lambda}\left(\varepsilon_{\beta \lambda \mid \gamma}-\varepsilon_{\beta \gamma \mid \lambda}\right)\right]=0, \\
e^{\alpha \lambda} e^{\beta \mu}\left(\kappa_{\alpha \beta} b_{\lambda \mu}-\varepsilon_{\alpha \beta \mid \lambda \mu}\right)=0
\end{gathered}
$$

are the three equations of compatibility for shells.

The distributed surface load $p$ is resolved in the directions of the base vectors $\mathbf{a}_{\alpha}$ and the normal vector $\mathbf{a}_{3}$; that is, $\mathbf{p}=$ $p^{\alpha} \mathbf{a}_{\alpha}+p^{3} \mathbf{a}_{3}$. If we write $\mathbf{N}^{\alpha}$ and $\mathbf{M}^{\alpha}$ in the form $\mathbf{N}^{\alpha}=N^{\alpha \beta} \mathbf{a}_{\beta}+$ $Q^{\alpha} \mathbf{a}_{3}, M^{\alpha}=M^{\alpha \beta} \widehat{\mathbf{a}}_{\beta}$, it can be seen that $N^{\alpha \beta}, Q^{\alpha}$, and $M^{\alpha \beta}$ are tensors, the types and orders of which are given by the positions and the numbers of indices. The quantities $N^{\alpha \beta}, Q^{\alpha}$, and $M^{\alpha \beta}$ are referred to as the complete contact forces and couples. Hence, the six scalar equations of equilibrium of the shell are as follows: $N^{\alpha \beta}{ }_{\mid \alpha}-b_{\alpha}^{\beta} Q^{\alpha}+p^{\beta}=0, Q^{\alpha}{ }_{\mid \alpha}+N^{\alpha \beta} b_{\alpha \beta}+p^{3}=$ $0, M_{\mid \alpha}^{\alpha \beta}-Q^{\beta}=0, e_{\alpha \beta}\left(N^{\alpha \beta}-b_{\lambda}^{\alpha} M^{\lambda \beta}\right)=0$. For a thin shell consisting of a homogeneous and isotropic elastic material, the constitutive equations for the effective contact equations have the form

$$
\begin{gathered}
N^{\alpha \beta}=\frac{E h}{1-v^{2}}\left[(1-v) a^{\alpha \lambda} a^{\beta \mu}+v a^{\alpha \beta} a^{\lambda \mu}\right] \varepsilon_{\lambda \mu}, \\
M^{\alpha \beta}=\frac{E h^{3}}{12\left(1-v^{2}\right)}\left[(1-v) a^{\alpha \lambda} a^{\beta \mu}+v a^{\alpha \beta} a^{\lambda \mu}\right] \kappa_{\lambda \mu} .
\end{gathered}
$$

The specific strain energy of the shell is given by

$$
e=\frac{1}{2}\left[N^{\alpha \beta} \varepsilon_{\alpha \beta}+M^{\alpha \beta} \kappa_{\alpha \beta}\right] \text {. }
$$

\section{Gradient Theory of Elasticity}

The implicit version of gradient elasticity, which combines both Eringen's stress-gradient and Aifantis' stable straingradient theory, can be summarized by the following gradient constitutive equation $\left(1-l_{d}^{2} \nabla^{2}\right) \sigma_{i j}=C_{i j k l}\left(1-l_{s}^{2} \nabla^{2}\right) \varepsilon_{k l}$. The stress gradient internal length may be set, under certain circumstances, equal to the internal length scale for dynamics $\left(l_{\sigma} \approx l_{d}\right)$. The strain gradient internal length is identified with the internal length scale for statics $\left(l_{\varepsilon} \approx l_{s}\right)$. The symbol $\sigma_{i j}$ denotes the stress tensor and the symbol $\varepsilon_{k l}$ denotes the strain tensor, while $C_{i j k l}$ is the linear elastic stiffness tensor. It is worth noting that the length scales for statics and dynamics are not equal in general. When the static length scale is zero, the theory is a special form of Aifantis' straingradient elasticity, and when the dynamics length scale is zero, the theory reduces to Eringen's stress-gradient elasticity theory. When both internal length scale parameters tend to zero, the theory becomes Hooke's law of classical elasticity. Identification and quantification of the internal lengths have been discussed in Askes and Aifantis [15], which is beyond the focus of this paper.

Due to the fact that $\left|l_{d}^{2} l_{s}^{2} \nabla^{2} \nabla^{2}\right| \sim 1$, the gradient elasticity constitutive relations can be approximated up to 2 nd order terms as follows: $\left(1+l_{s}^{2} \nabla^{2}-l_{d}^{2} \nabla^{2}\right) \sigma_{i j}=C_{i j k l} \varepsilon_{k l}$, or the alternative form $\sigma_{i j}=C_{i j k l}\left(1+l_{d}^{2} \nabla^{2}-l_{s}^{2} \nabla^{2}\right) \varepsilon_{k l}$. These relations will be used in the sequel to derive robust approximate but quite general results appropriate for all formulations.

\section{Formulation of Linear Gradient Shell Theory Based on $\left(1-l_{d}^{2} \nabla^{2}\right) \sigma_{i j}=C_{i j k l}\left(1-l_{s}^{2} \nabla^{2}\right) \varepsilon_{k l}$}

Once we adopt the constitutive equation of implicit gradient elasticity $\left(1-l_{d}^{2} \nabla^{2}\right) \sigma_{i j}=C_{i j k l}\left(1-l_{s}^{2} \nabla^{2}\right) \varepsilon_{k l}$, then the corresponding theory for gradient shells can be easily formulated. For orthogonal coordinates, we have the two-dimensional Laplace operator

$\nabla^{2}(\cdots)=\frac{1}{A_{1} A_{2}}\left\{\frac{\partial}{\partial \alpha_{1}}\left[\frac{A_{2}}{A_{1}} \frac{\partial(\cdots)}{\partial \alpha_{1}}\right]+\frac{\partial}{\partial \alpha_{2}}\left[\frac{A_{1}}{A_{2}} \frac{\partial(\cdots)}{\partial \alpha_{2}}\right]\right\}$

where $A_{i}$ denote Láme parameters of the mid-surface of the shell.

For a thin shell consisting of a homogeneous, isotropic elastic material, the constitutive equations for the effective contact equations have the constitutive equation (3).

Then, their gradient counterparts read as follows:

$$
\begin{aligned}
& \left(1-l_{d}^{2} \nabla^{2}\right) N^{\alpha \beta} \\
& =\frac{E h}{1-v^{2}}\left[(1-v) a^{\alpha \lambda} a^{\beta \mu}+v a^{\alpha \beta} a^{\lambda \mu}\right]\left(1-l_{s}^{2} \nabla^{2}\right) \varepsilon_{\lambda \mu}, \\
& \left(1-l_{d}^{2} \nabla^{2}\right) M^{\alpha \beta} \\
& =\frac{E h^{3}}{12\left(1-v^{2}\right)}\left[(1-v) a^{\alpha \lambda} a^{\beta \mu}+v a^{\alpha \beta} a^{\lambda \mu}\right]\left(1-l_{s}^{2} \nabla^{2}\right) \kappa_{\lambda \mu} .
\end{aligned}
$$

The corresponding balance equations read

$$
\begin{gathered}
\left(1-l_{s}^{2} \nabla^{2}\right)\left[{ }_{\mid \alpha}^{\alpha \beta}-b_{\alpha}^{\beta} Q^{\alpha}\right]+\left(1-l_{d}^{2} \nabla^{2}\right) p^{\beta}=0, \\
\left(1-l_{s}^{2} \nabla^{2}\right)\left[Q_{\mid \alpha}^{\alpha}+N^{\alpha \beta} b_{\alpha \beta}\right]+\left(1-l_{d}^{2} \nabla^{2}\right) p^{3}=0, \\
M_{\mid \alpha}^{\alpha \beta}-Q^{\beta}=0 .
\end{gathered}
$$

The specific strain energy of the shell is given by $W=\left[1+\left(l_{d}^{2}-\right.\right.$ $\left.\left.l_{s}^{2}\right) \nabla^{2}\right] e$, where $e$ denotes the classical strain energy density of the shell; that is,

$$
\begin{aligned}
e= & \frac{1}{2} \frac{E h}{1-v^{2}}\left[(1-v) \varepsilon^{\alpha \beta} \varepsilon_{\alpha \beta}+v \varepsilon_{\alpha}^{\alpha} \varepsilon_{\beta}^{\beta}\right] \\
& +\frac{E h^{3}}{24\left(1-v^{2}\right)}\left[(1-v) \kappa^{\alpha \beta} \kappa_{\alpha \beta}+v \kappa_{\alpha}^{\alpha} \kappa_{\beta}^{\beta}\right] .
\end{aligned}
$$




\section{Formulation of Linear Gradient Shell \\ Theory Based on $\sigma_{i j} \cong C_{i j k l}\left(1+l_{d}^{2} \nabla^{2}-l_{s}^{2} \nabla^{2}\right) \varepsilon_{k l}$}

The appropriate gradient constitutive equations for this shell theory take the form

$$
\begin{aligned}
N^{\alpha \beta}= & \frac{E h}{1-v^{2}}\left[(1-v) a^{\alpha \lambda} a^{\beta \mu}+v a^{\alpha \beta} a^{\lambda \mu}\right] \\
& \times\left(1+l_{d}^{2} \nabla^{2}-l_{s}^{2} \nabla^{2}\right) \varepsilon_{\lambda \mu}, \\
M^{\alpha \beta}= & \frac{E h^{3}}{12\left(1-v^{2}\right)}\left[(1-v) a^{\alpha \lambda} a^{\beta \mu}+v a^{\alpha \beta} a^{\lambda \mu}\right] \\
& \times\left(1+l_{d}^{2} \nabla^{2}-l_{s}^{2} \nabla^{2}\right) \kappa_{\lambda \mu},
\end{aligned}
$$

and the balance equations read

$$
\begin{gathered}
\left(1+l_{d}^{2} \nabla^{2}-l_{s}^{2} \nabla^{2}\right)\left[{ }^{\alpha \beta}{ }_{\mid \alpha}-b_{\alpha}^{\beta} Q^{\alpha}\right]+p^{\beta}=0, \\
\left(1+l_{d}^{2} \nabla^{2}-l_{s}^{2} \nabla^{2}\right)\left[Q_{\mid \alpha}^{\alpha}+N^{\alpha \beta} b_{\alpha \beta}\right]+p^{3}=0, \\
Q^{\beta}=M_{\mid \alpha}^{\alpha \beta} .
\end{gathered}
$$

\section{Formulation of Linear Gradient Shell$$
\text { Theory Based on }\left(1+l_{s}^{2} \nabla^{2}-l_{d}^{2} \nabla^{2}\right) \sigma_{i j} \cong C_{i j k l} \varepsilon_{k l}
$$

The appropriate gradient constitutive equations for this shell theory take the form

$$
\begin{aligned}
& \left(1-l_{d}^{2} \nabla^{2}+l_{s}^{2} \nabla^{2}\right) N^{\alpha \beta} \\
& =\frac{E h}{1-v^{2}}\left[(1-v) a^{\alpha \lambda} a^{\beta \mu}+v a^{\alpha \beta} a^{\lambda \mu}\right] \varepsilon_{\lambda \mu}, \\
& \left(1-l_{d}^{2} \nabla^{2}+l_{s}^{2} \nabla^{2}\right) M^{\alpha \beta} \\
& =\frac{E h^{3}}{12\left(1-v^{2}\right)}\left[(1-v) a^{\alpha \lambda} a^{\beta \mu}+v a^{\alpha \beta} a^{\lambda \mu}\right] \kappa_{\lambda \mu},
\end{aligned}
$$

and the balance equations read

$$
\begin{gathered}
{\left[N_{\mid \alpha}^{\alpha \beta}-b_{\alpha}^{\beta} Q^{\alpha}\right]+\left(1-l_{d}^{2} \nabla^{2}+l_{s}^{2} \nabla^{2}\right) p^{\beta}=0} \\
{\left[Q_{\mid \alpha}^{\alpha}+N^{\alpha \beta} b_{\alpha \beta}\right]+\left(1-l_{d}^{2} \nabla^{2}+l_{s}^{2} \nabla^{2}\right) p^{3}=0} \\
Q^{\beta}=M_{\mid \alpha}^{\alpha \beta} .
\end{gathered}
$$

\section{Conclusions}

Various gradient (nano)shell models can be easily formulated by simply replacing the classical constitutive equations of classical elasticity with the constitutive equation of the implicit gradient elasticity $\left(1-l_{d}^{2} \nabla^{2}\right) \sigma_{i j}=C_{i j k l}\left(1-l_{s}^{2} \nabla^{2}\right) \varepsilon_{k l}$ and its approximate versions of strain gradient $\sigma_{i j}=C_{i j k l}(1+$ $\left.l_{d}^{2} \nabla^{2}-l_{s}^{2} \nabla^{2}\right) \varepsilon_{k l}$ and stress gradient $\left(1+l_{s}^{2} \nabla^{2}-l_{d}^{2} \nabla^{2}\right) \sigma_{i j}=$ $C_{i j k l} \varepsilon_{k l}$ counterparts. All formulations can also be extended in an analogous manner, to geometrically nonlinear theory of gradient shells [16].

\section{Conflict of Interests}

The authors declare that there is no conflict of interests regarding the publication of this paper.

\section{Acknowledgment}

E. C. Aifantis acknowledges support of GSRT under grants Thales Intermonu and ERC-13, as well as a HiCi grant from KAU.

\section{References}

[1] A. Cauchy, "Memoire sur les systemes isotropes de points materiels," in Oeuvres completes - Tome II, 1st Serie, pp. 351-386, Gauthier-Villars, Paris, France, 1850, reprint 1908.

[2] A. Cauchy, "Memoire sur les vibrations d'un double systeme de molecules et de l'ether continu dans un corps cristallise," in Oeuvres completes-Tome II, 1st Serie, pp. 338-350, GauthierVillars, Paris, France, 1850, reprint 1908.

[3] E. Cosserat and F. Cosserat, Theorie des Corps Deformables, Hermann et Fils, Paris, France, 1909.

[4] R. D. Mindlin, "Micro-structure in linear elasticity," Archive for Rational Mechanics and Analysis, vol. 16, no. 1, pp. 51-78, 1964.

[5] R. D. Mindlin, "Second gradient of strain and surface-tension in linear elasticity," International Journal of Solids and Structures, vol. 1, no. 4, pp. 417-438, 1965.

[6] A. C. Eringen, "Nonlocal continuum theory for dislocations and fracture," in The Mechanics of Dislocations, E. C. Aifantis and J. P. Hirth, Eds., pp. 101-110, ASM, Metals Park, Ohio, USA, 1985.

[7] A. C. Eringen, "Vistas of nonlocal continuum physics," International Journal of Engineering Science, vol. 30, no. 10, pp. 1551$1565,1992$.

[8] A. C. Eringen, "On differential equations of nonlocal elasticity and solutions of screw dislocation and surface waves," Journal of Applied Physics, vol. 54, no. 9, pp. 4703-4710, 1983.

[9] A. C. Eringen, Nonlocal Continuum Field Theories, Springer, 2012.

[10] E. C. Aifantis, "On the role of gradients in the localization of deformation and fracture," International Journal of Engineering Science, vol. 30, no. 10, pp. 1279-1299, 1992.

[11] E. C. Aifantis, "On the gradient approach: relation to Eringen's nonlocal theory," International Journal of Engineering Science, vol. 49, no. 12, pp. 1367-1377, 2011.

[12] E. C. Aifantis, "Update on a class of gradient theories," Mechanics of Materials, vol. 35, pp. 259-280, 2009.

[13] H. Mollmann, Introduction to the Theory of Thin Shells, John Wiley \& Sons, New York, NY, USA, 1981.

[14] A. E. H. Love, A Treatise on the Mathematical Theory of Elasticity, Cambridge University Press, Cambridge, UK, 4th edition, 1927.

[15] H. Askes and E. C. Aifantis, "Gradient elasticity in statics and dynamics: an overview of formulations, length scale identification procedures, finite element implementations and new results," International Journal of Solids and Structures, vol. 48, no. 13, pp. 1962-1990, 2011.

[16] B. Sun, "Gradient theory of nanoplates and nanoshells," in Proceedings of the 12th Annual SPE Automotive Composites Conference and Exhibition (ACCE '12), Beijing, China, 2012. 

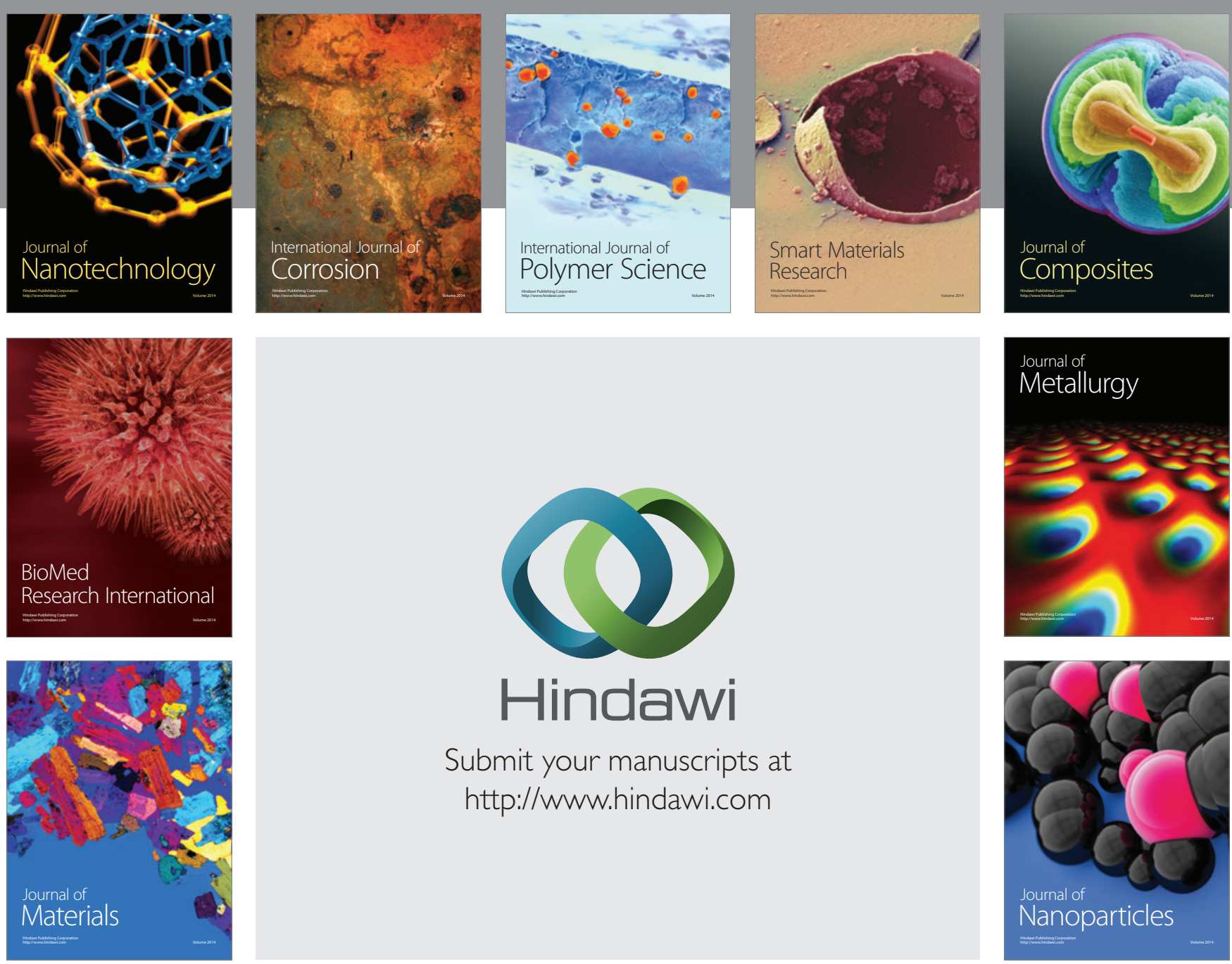

Submit your manuscripts at http://www.hindawi.com
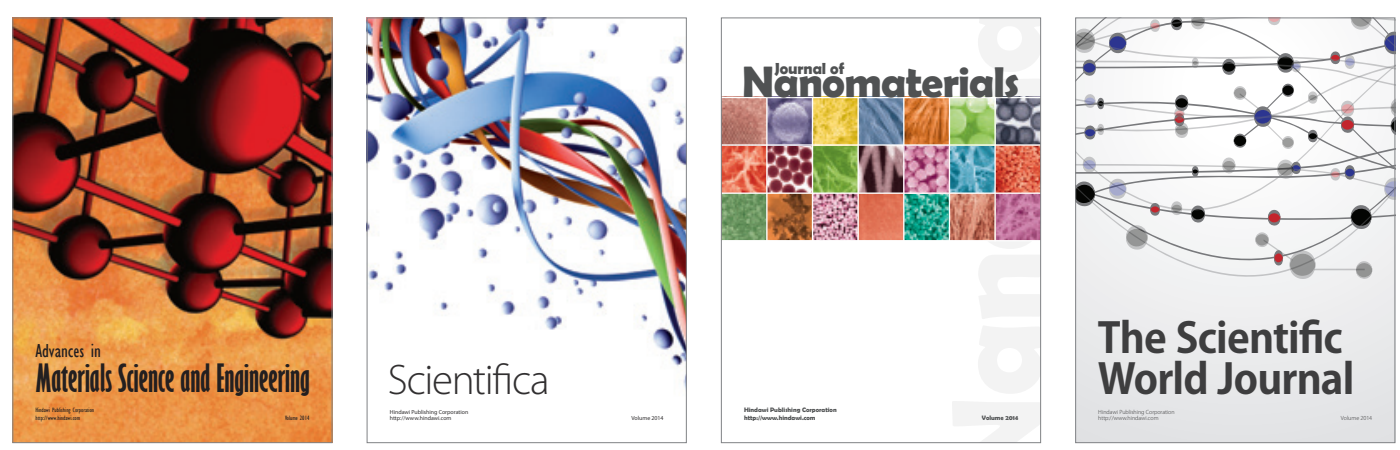

\section{The Scientific World Journal}
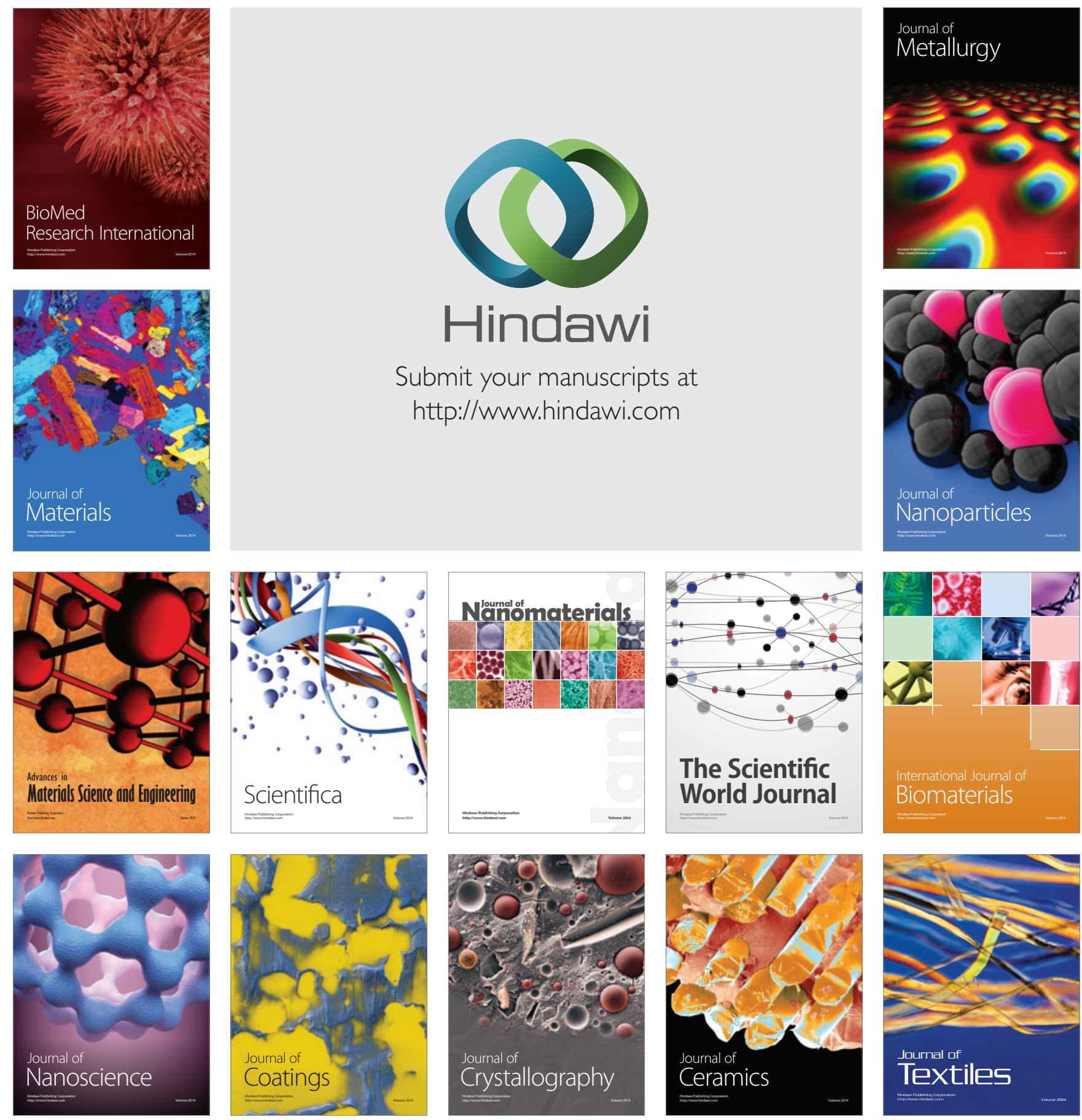